\title{
Histopathological analysis of rat mesentery as a method for evaluating neutrophil migration: differential effects of dexamethasone and pertussis toxin
}

\footnotetext{
G.A.C. Brito ${ }^{2}$, J.L.A.A. Falcão ${ }^{1}$, S.N.R. Saraiva ${ }^{2}$ A.A.M. Limal,

C.A. Flores ${ }^{1}$ and R.A. Ribeiro ${ }^{1}$
}

\author{
${ }^{1}$ Departamento de Fisiologia e Farmacologia, Unidade de Pesquisa Clínica, \\ and ${ }^{2}$ D epartamento de M orfologia, Faculdade de Medicina, \\ Universidade Federal do Ceará, Fortaleza, CE, Brasil
}

\section{Correspondence \\ R.A. Ribeiro \\ Departamento de Fisiologia \\ e Farmacologia, CCS, UFCE \\ Rua Coronel Nunes de Melo, 1127 \\ 60430-270 Fortaleza, CE \\ Brasil \\ Fax: + 55-85-243-9333 \\ E-mail: gerly@fortalnet.com.br}

Research supported by CN Pq (No. 523808/96-8) and FUNCAP (No. SOS 092/94).

Received August 26, 1997 Accepted June 22, 1998

\section{Abstract}

In the present study, histopathological analysis of rat mesentery was used to quantify the effect of two anti-inflammatory agents, dexamethasone (Dex) and pertussis toxin (Ptx), on leukocyte migration. The intravenous injection of Dex (1 mg/kg) and Ptx (1,200 ng) $1 \mathrm{~h}$ prior to the intraperitoneal injection of the inflammatory stimuli lipopolysaccharide (LPS) or formyl-methionyl-leucyl-phenylalanine (fMLP) significantly reduced the neutrophil diapedesis (LPS: Ptx $=0.86 \pm 0.19$ and Dex $=0.35 \pm 0.13$ vs saline $(S)=2.85 \pm 0.59$; $\mathrm{fMLP}: \mathrm{Ptx}=0.43 \pm$ 0.09 and Dex $0.01 \pm 0.01$ vs $\mathrm{S}=1.08 \pm 0.15$ neutrophil diapedesis/ field) and infiltration (LPS: Ptx $=6.29 \pm 1.4$ and Dex $=3.06 \pm 0.76 v \mathrm{~s}$ $\mathrm{S}=15.94 \pm 3.97$; fMLP: Ptx $=3.85 \pm 0.56$ and Dex $=0.40 \pm 0.16 v s$ $\mathrm{S}=7.15 \pm 1.17$ neutrophils/field) induced by the two agonists in the rat mesentery. The inhibitory effect of Dex and Ptx was clearly visible in the fields nearest the venule (up to $200 \mu \mathrm{m}$ ), demonstrating that these anti-inflammatory agents act preferentially in the transmigration of neutrophils from the vascular lumen into the interstitial space, but not in cell movement in response to a haptotactic gradient. The mesentery of rats pretreated with Dex showed a decreased number of neutrophils within the venules (LPS: $\operatorname{Dex}=1.50 \pm 0.38$ vs $\mathrm{S}=4.20 \pm 1.01$; fMLP: Dex $=0.25 \pm 0.11$ vs $\mathrm{S}=2.20 \pm 0.34$ neutrophils in the lumen/field), suggesting that this inhibitor may be acting at a step that precedes neutrophil arrival in the inflamed tissue. In contrast to that observed with Dex treatment, the number of neutrophils found in mesenteric venules was significantly elevated in animals pretreated with Ptx (LPS: Ptx $=9.85 \pm 2.25$ vs $\mathrm{S}=4.20 \pm 1.01$; fMLP: $\mathrm{Ptx}=4.66 \pm 1.24$ vs $\mathrm{S}=2.20 \pm 0.34$ neutrophils in the lumen/field). This discrepancy shows that Ptx and Dex act via different mechanisms and suggests that Ptx prevents locomotion of neutrophils from the vascular lumen to the interstitial space. In conclusion, the method described here is useful for quantifying the inflammatory and anti-inflammatory effect of different substances. The advantage of this histopathological approach is that it provides additional information about the steps involved in leucocyte migration.

\section{Key words}

- Neutrophil migration

- Pertussis toxin

- LPS

- Dexamethasone

- Mesentery

- Histopathological approach 


\section{Introduction}

The migration of specific leukocyte subsets from the microvascular lumen to extravascular tissue is a characteristic feature of the inflammatory response. Such leukocytes function as the primary line of host defense in the destruction of microorganisms and in the initiation of tissue repair. This process is initiated by the extravascular generation of chemoattractants $(1,2)$ and by the formation of weak reversible adhesive interactions between leukocytes and vascular endothelial cells. This binding is mediated by selectins, and although of relatively low affinity, is sufficient to serve as a biological brake which quickly decelerates leukocytes by making them roll on endothelial cells. During rolling, leukocytes may be activated by chemoattractants, with a consequent greatly increased affinity of their $\beta_{2}$ integrin adhesion receptors for ligands on activated endothelium. A chemotactic signal present outside the venule induces leukocytes to squeeze between the endothelial cells of the venule and to migrate to the site of inflammation $(3,4)$.

Although leukocytes are necessary for host defense against infection, an excessive infiltration of these cells into the inflammatory site can cause tissue damage. Thus, efforts have been made to understand this process, and to develop substances that can interfere with specific steps of leukocyte recruitment.

The mesentery is a translucent tissue that allows direct viewing of the microcirculation without microtomy (5). This tissue can be easily inflamed by the injection of various stimuli into the peritoneal cavity, and has been extensively used as a model for anti-inflammatory drug screening. The easiest means of quantifying leukocyte infiltration is to perform total and differential cell counts in peritoneal exudates (6). However, using this procedure, little information is obtained about the mechanisms involved in leukocyte recruitment into the inflamed mesentery. The technique of in- travital microscopy was introduced to overcome this deficiency and to allow the gathering of important information on the contribution of adhesion molecules to the mechanisms that mediate the passage of leukocytes across the vessel wall in vivo $(3,7)$. The principal disadvantages of this method are its level of sophistication and elevated cost, since it requires a good recording setup (microscope, camera, video monitor) and computer facilities (software, printer).

The objetive of the present study was to develop a simpler and less expensive method for the analysis and quantification of neutrophil migration into inflamed peritoneal cavities based on histopathological features of the rat mesentery. Throughout this study, we used lipopolysaccharide (LPS) and formylmethionyl-leucyl-phenylalanine (fMLP) as the pro-inflammatory stimuli and dexamethasone (Dex) (8) and pertussis toxin (Ptx) from Bordetella pertussis (9) as inhibitors of neutrophil migration. We have recently demonstrated that the inhibitory activity of pertussis toxin depends on the ADP-ribosylating activity of its A protomer on $\mathrm{G}$ proteins (10).

\section{Material and Methods}

\section{Animals}

Male Wistar rats (150-200 g) from the animal colony of the Federal University of Ceará were used throughout the study. The animals received water and food ad libitum.

\section{Drugs and toxins}

Lipopolysaccharide from Escherichia coli 0111:B4 (Difco, Detroit, MI) and N-formylmethionyl-leucyl-phenylalanine (Sigma Chemical Co., St. Louis, MO) were used as neutrophil chemotactic stimuli. Dexamethasone acetate (Prodrome, Campinas, SP, Brasil) was used as an anti-inflammatory agent. Prior to use, the drugs were diluted from stock solutions in sterile $0.9 \% \mathrm{NaCl}$. 
Pertussis toxin was purified from the supernatants of $B$. pertussis 165 cultures by hydroxylapatite chromatography and fetuin affinity chromatography (11). Ptx was a generous gift from Dr. Erik Hewlett (University of Virginia, VA, USA).

\section{Experimental protocols}

LPS (200 ng/cavity) or fMLP (44 ng/ cavity) was injected intraperitoneally ( $i p ; 1$ $\mathrm{ml}$ ) into rats pretreated intravenously (iv) $1 \mathrm{~h}$ earlier with Ptx (1,200 ng), Dex (1 mg/kg) or saline $(0.2 \mathrm{ml})$.

The animals were sacrificed 2,3 or $4 \mathrm{~h}$ after the ip injection of LPS and $4 \mathrm{~h}$ after fMLP, and the peritoneal cells harvested by washing the cavities with $10 \mathrm{ml}$ of PBS containing $5 \mathrm{U}$ heparin $/ \mathrm{ml}$. Total and differential cell counts were performed as described elsewhere (6). The results are reported as number of neutrophils/ml. After harvesting the exudate, the peritoneal cavity was opened, the mesentery removed and fixed in $10 \%$ neutral buffered formalin. After $48 \mathrm{~h}$, the tissue was sectioned into small pieces and mounted on albumin-coated glass slides. Twenty-four hours later the histological sections were stained with hematoxylin and eosin. Quantitative histological analysis was performed by light microscopy using a 1000X-immersion objective. The extent of neutrophil infiltration was quantified in five consecutive microscopic fields on both sides of the luminal and extraluminal sides. The number of neutrophils inside the vessel and the number of cells in diapedesis (neutrophils passing between the venule endothelial cells, i.e., transmigration) were obtained in five fields along the venule, in mesentery of animals sacrificed $4 \mathrm{~h}$ after the inflammatory stimuli. The results are reported as the mean \pm SEM of the number of neutrophils/field.

\section{Statistical analysis}

The statistical significance of the differ- ences among groups was determined by ANOVA followed by the Tukey test. Regression analysis was performed to determine the 95\% confidence interval and the linear correlation coefficient (r). P values less than $5 \%$ were considered to indicate statistical significance.

\section{Results}

\section{LPS- and fMLP-induced neutrophil infiltration into rat mesenteric tissue}

Ptx (1,200 ng/rat) and Dex (0.2 mg/rat) injected $i v 1 \mathrm{~h}$ before LPS (200 ng) or fMLP $(44 \mathrm{ng})$ significantly $(\mathrm{P}<0.05)$ reduced the mesenteric neutrophil infiltration induced by both inflammatory stimuli (Figure 1A and $\mathrm{C}$ ). The reduced neutrophil infiltration could be seen in the first $200 \mu \mathrm{m}$ close to the venule $(\mathrm{P}<0.05$; Figure $1 \mathrm{~B}$ and $\mathrm{D})$. The effect of Dex or Ptx on LPS-induced neutrophil infiltration is illustrated in Figure 2. There was a clear reduction in the number of neutrophils in the mesentery of animals pretreated with Ptx (C and D) or Dex (E and F) when compared to controls (A and B). A similar response was observed with fMLP (data not shown). Regression analysis showed that there was a positive correlation $(\mathrm{r}=$ 0.77 ) between the number of infiltrated neutrophils in the mesentery and the number of these cells present in the peritoneal washes 4 $\mathrm{h}$ after the injection of LPS. The 95\% confidence interval of the correlation coefficient for the population was different from zero, indicating that there was a linear correlation between the methods used (Figure 3 ). When the animals were sacrificed 2 or $3 \mathrm{~h}$ after the injection of LPS, the correlation coefficient between the methods was $r=0.74$ and $r=$ 0.68 , respectively (data not shown).

Time course of neutrophil infiltration induced by LPS into rat mesenteric tissue

Ptx (1,200 ng/rat) and Dex (0.2 mg/rat) 
injected $i v 1 \mathrm{~h}$ before LPS (200 ng) significantly $(\mathrm{P}<0.05)$ reduced the mesenteric neutrophil infiltration in the $2 \mathrm{nd}, 3 \mathrm{rd}$ and 4 th $\mathrm{h}$ after the inflammatory stimuli (Figure 4). The peak of neutrophil infiltration in the mesentery was seen at the 3rd h (Figure 4), whereas the highest counts of neutrophil in peritoneal wash were obtained $4 \mathrm{~h}$ after the inflammatory stimuli (data not shown).

\section{Neutrophil diapedesis induced by LPS and fMLP in mesenteric venules}

Ptx $(1,200 \mathrm{ng} / \mathrm{rat})$ or Dex $(0.2 \mathrm{mg} / \mathrm{rat})$
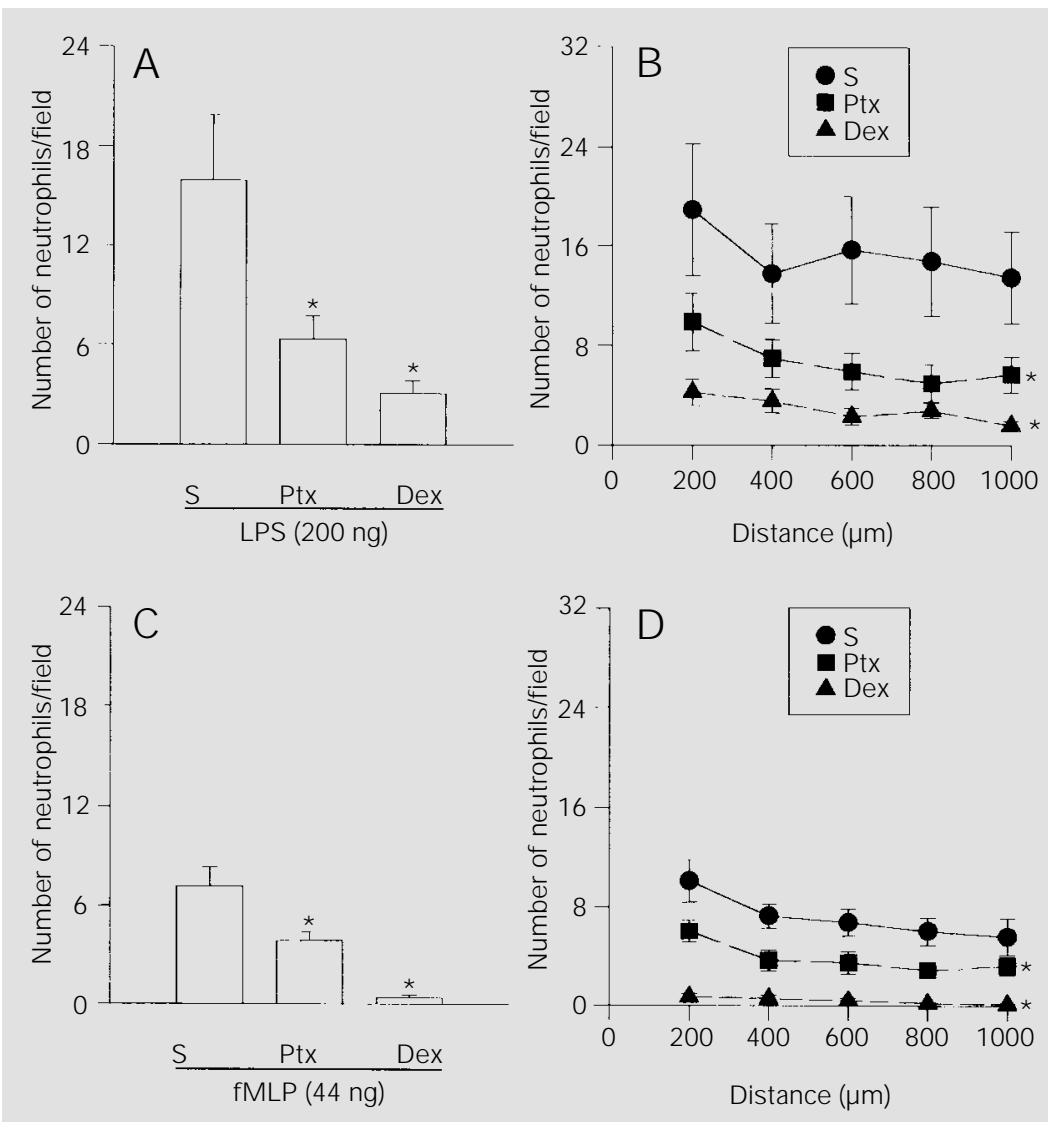

Figure 1 - Pertussis toxin (Ptx) and dexamethasone (Dex) inhibit LPS- or fMLP-induced neutrophil migration into the rat mesentery. Ptx $(1,200 \mathrm{ng} / \mathrm{rat})$ and Dex $(0.2 \mathrm{mg} / \mathrm{rat})$ were injected iv $1 \mathrm{~h}$ before the ip administration of LPS ( $200 \mathrm{ng} /$ cavity; A and B) or fMLP ( $44 \mathrm{ng} /$ cavity; $C$ and $D$ ). The rats were sacrificed $4 \mathrm{~h}$ after injection of the inflammatory stimuli. The neutrophils were counted in five consecutive fields on both sides of a venule perpendicular axis. The results are reported as the mean \pm SEM of the total number of neutrophils/field ( $A$ and $C$ ) or number of neutrophils/field as a function of the distance from the venule ( $B$ and $D$ ). Six rats were used per treatment. $* \mathrm{P}<0.05$ compared to the group that received saline $(\mathrm{S})$ iv at all points. (ANOVA and Tukey test). administered iv $1 \mathrm{~h}$ before LPS (200 $\mathrm{ng}$ ) or fMLP $(44 \mathrm{ng})$ significantly reduced $(\mathrm{P}<0.05)$ the number of neutrophils in diapedesis across the mesenteric venule wall (Figure $5 \mathrm{~A}$ and $\mathrm{B})$.

\section{Neutrophils in the lumen of the mesenteric venule}

The number of neutrophils inside the mesenteric venules was significantly increased $(\mathrm{P}<0.05)$ by Ptx $(1,200 \mathrm{ng} / \mathrm{rat})$ injected $i v 1 \mathrm{~h}$ before LPS (200 ng) or fMLP (44 ng) challenge (Figure 6A and B). On the other hand, Dex $(0.2 \mathrm{mg} / \mathrm{rat})$ administered $i v$ $1 \mathrm{~h}$ before LPS or fMLP (in the same doses) significantly reduced $(\mathrm{P}<0.05)$ the number of neutrophils inside the mesenteric venules when compared to saline-treated rats (Figure $6 \mathrm{~A}$ and $\mathrm{B})$.

\section{Discussion}

The histopathological method presented here proved to be useful for quantifying leukocyte migration and the effect of antiinflammatory agents on this phenomenon. Our results confirmed the inhibitory effect of Dex (12) and Ptx (10) on neutrophil migration induced by inflammatory stimuli. Rats treated with these agents showed a significant reduction in the neutrophil infiltration into inflamed mesentery induced by fMLP, a direct chemotactic agent, and LPS, a substance that induces neutrophil recruitment by an indirect mechanism dependent on peritoneal resident cells (13).

Dexamethasone reduced the neutrophil infiltration induced by both chemotactic stimuli. The inhibitory effect of Dex and Ptx was particularly visible in the fields nearest the venule (within a distance of up to 200 $\mu \mathrm{m})$, thus demonstrating that these anti-inflammatory agents act preferentially on the migration of neutrophils from the vascular lumen into the interstitial space, rather than on the movement of cells in response to a 

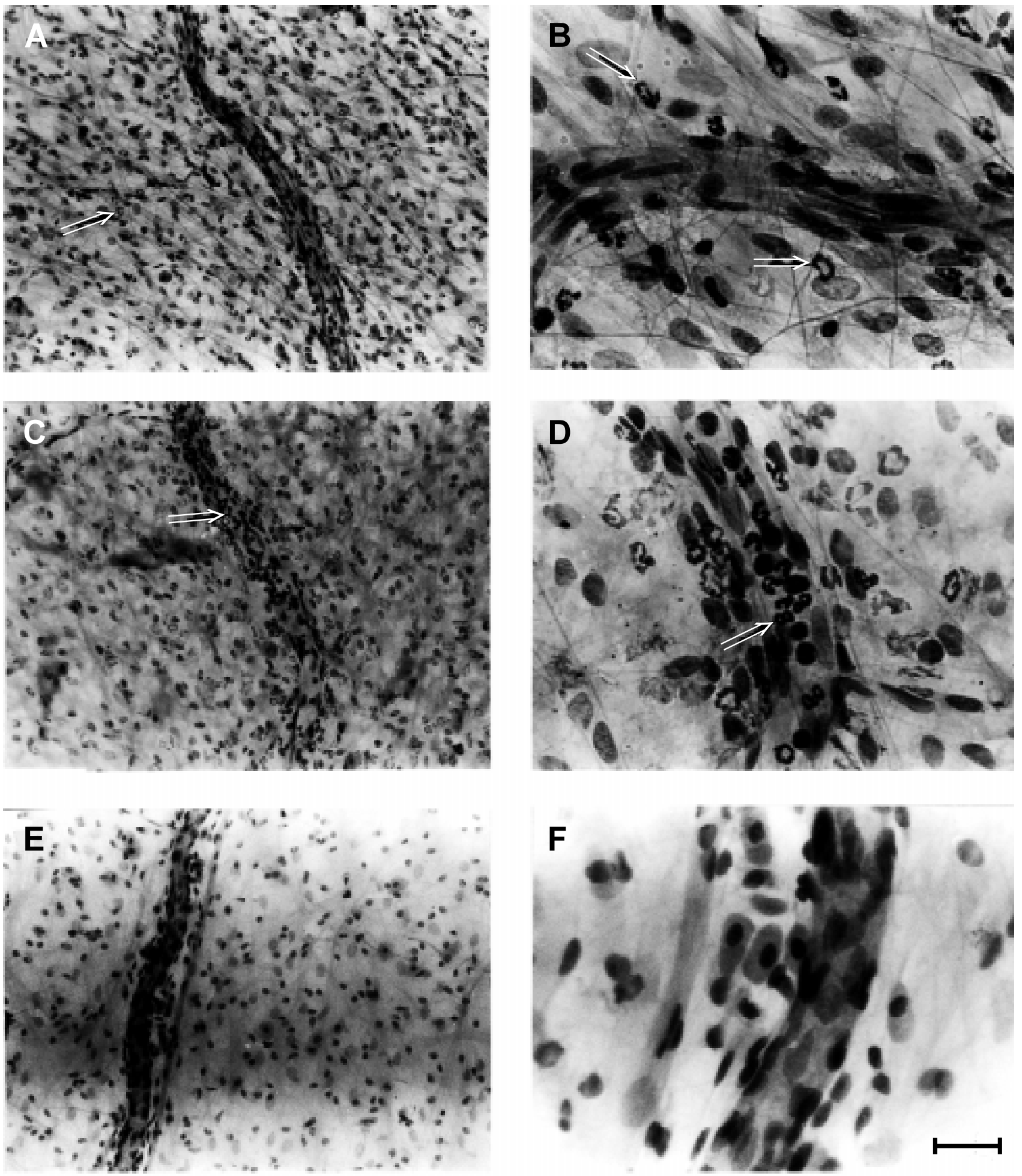

Figure 2 - Histological aspect of inflamed rat mesentery $4 \mathrm{~h}$ after the ip injection of LPS ( $200 \mathrm{ng} / \mathrm{cavity}$ ) and iv pretreated ( $1 \mathrm{~h}$ earlier) with sterile saline ( $A$ and $B$ ), pertussis toxin (Ptx; 1,200 ng/animal; $C$ and D) or dexamethasone (Dex; 0.2 mg/animal; $E$ and F). The neutrophil infil trate in the Ptx-and Dextreated rats was clearly less intense than that observed in the saline-treated group. In Ptx-treated rats, the neutrophils were retained in the lumen of the blood vessels. Arrows are pointing to neutrophils. Hematoxylin and eosin stain; bar $=100 \mu \mathrm{m}$ for A, C, and E, and bar $=25 \mu \mathrm{m}$ for B, D, and F. 
Figure 3 - The correlation between the histopathological method and conventional leukocyte counts in peritoneal exudate $4 \mathrm{~h}$ after the injection of LPS. The straight line represents the correlation index between these methods ( $r=0.77)$. The dashed lines represent the confidence interval $(P<0.05)$.
Figure 4 - Time course of LPSinduced neutrophil infiltration into rat mesenteric tissue. Pertussis toxin (Ptx; 1,200 ng/rat) or dexamethasone (Dex; $0.2 \mathrm{mg} /$ rat) was injected iv $1 \mathrm{~h}$ before the ip administration of LPS (200 ng/cavity). The rats were sacrificed 2, 3 and $4 \mathrm{~h}$ after injection of the inflammatory stimuli. The neutrophils were counted in five consecutive fields on both sides of a venule perpendicular axis. The results are reported as the mean \pm SEM of the total number of neutrophils/field. Six rats were used per treatment. $* \mathrm{P}<0.05$ compared to the group that received saline (S) iv at all times (ANOVA and Tukey test).
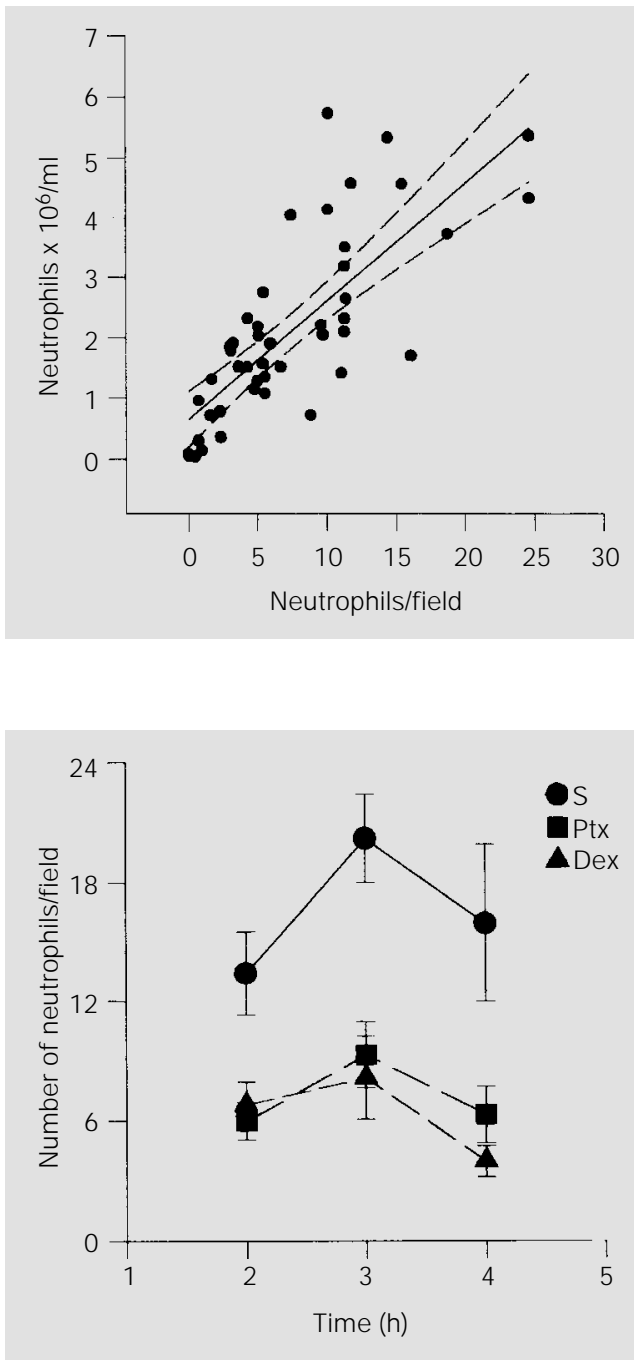

haptotactic gradient. This hypothesis is supported by two observations: a) at distances of $200 \mu \mathrm{m}$ from the venule, the curves for cell migration in the control and treated groups were almost parallel, and b) the number of neutrophils undergoing diapedesis in the mesenteric venules was significantly reduced in both Ptx- and Dex-treated rats.

It has been proposed by Katori et al. (12) that at least five sequential steps are involved in the extravasation of leukocytes from the vascular lumen into the interstitial space following the administration of chemoattractants: 1) rolling, 2) adhesion to the inner surface of the venules, 3) passage through endothelial gaps, 4) accumulation in the space between the endothelial cells and the basement membrane of pericytes, and 5) penetration of the pericyte basement membrane to reach the interstitial space. All of these steps can be triggered or modulated by chemotactic mediators, cytokines and interactions between leukocytes and endothelial cells through the expression of cell adhesion molecules (14). Dexamethasone is a potent anti-inflammatory agent with a well-known inhibitory action on neutrophil migration. Like other glucocorticoids, Dex induces the synthesis of lipocortin that inhibits the enzyme phospholipase $\mathrm{A}_{2}$, thereby preventing the generation of prostaglandins, leukotrienes and PAF which can contribute to the recruitment of leukocytes to the inflammatory site $(15,16)$. In addition, glucocorticoids inhibit the synthesis and activity of cytokines with chemotactic properties, including IL-1 $(17,18)$ and TNF- $\alpha(19,20)$, and their ability to induce the expression of adhesion molecules such as E-selectin, ICAM-1 (21) and VCAM-1 (22).

Our results show that the mesentery of rats pretreated with Dex had a lower number of neutrophils within the venule. However, this was not related to a possible neutropenic effect of the corticoid (data not shown). Indeed, acute treatment with corticoids causes neutrophilia (23). Thus, we suggest that Dex acts at a step that precedes neutrophil arrival in the inflamed tissue by inhibiting production and/or activity of chemotactic agents released by stimulated resident cells. Inhibition of chemotactic activity would explain the inhibitory effect of Dex on fMLP since this agent acts independently on the release of other chemotactic factors from peritoneal resident cells (24). In agreement with others (25), we believe that the inhibition of neutrophil-endothelium adhesion is the main mechanism for the anti-inflammatory effect of glucocorticoids. Such an action can explain the reductions in diapedesis and neutrophil infiltration observed in Dex-treated rats. On the other hand, some in vivo studies have 

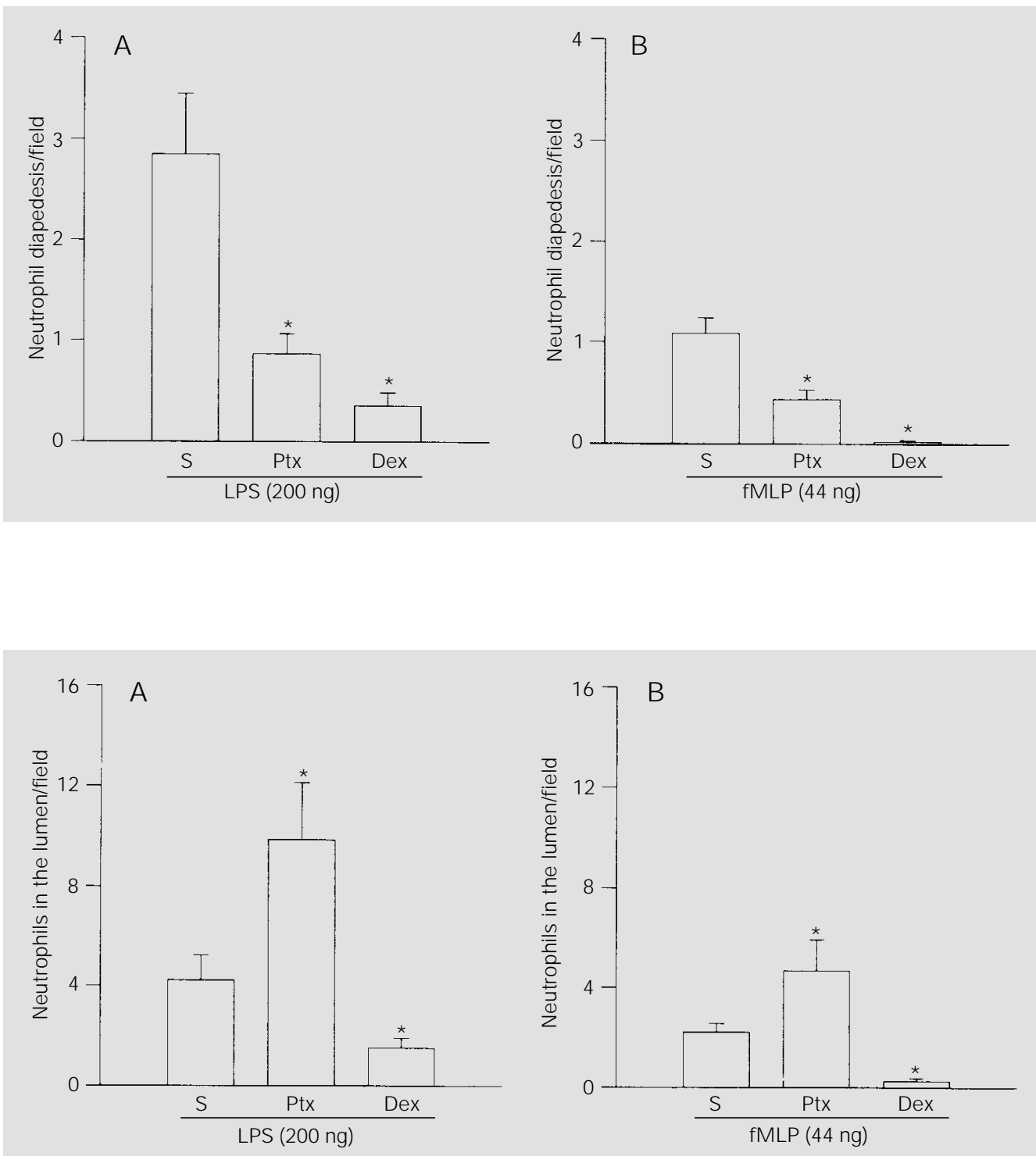

shown that Dex does not inhibit neutrophil rolling or adhesion to venular endothelium and does not inhibit neutrophil penetration through endothelial cell junctions $(12,26)$.

Ptx reduced the number of cells in diapedesis and the neutrophil infiltration into inflamed mesentery. In contrast to Dex, the number of neutrophils in the lumen of mesenteric venules was significantly increased in rats pretreated with this toxin. This divergence indicates that Ptx and Dex act via different mechanisms. The retention of neutrophils in the lumen of mesenteric blood

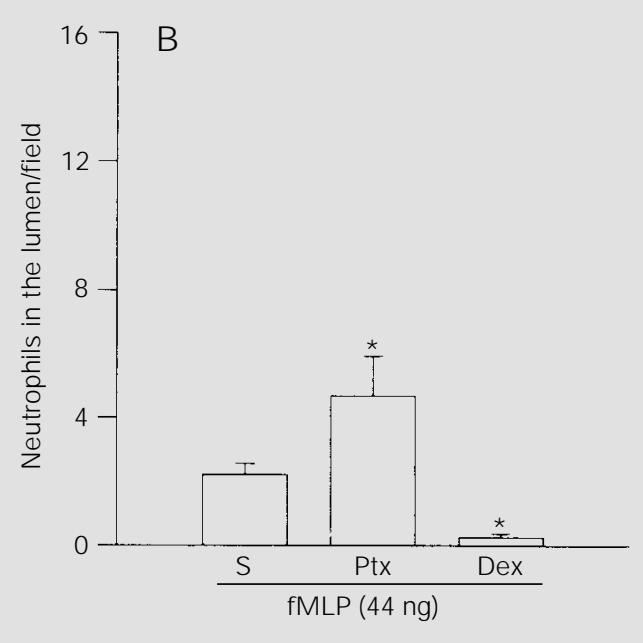

vessels suggests that Ptx prevents neutrophil locomotion from the vascular lumen into the interstitial space. It has been demonstrated that the lectin domains of two Ptx subunits share amino acid sequence similarities with the lectin domains of the eukaryotic selectin family (27). During the inflammatory process, selectins appear on endothelial cells and promote the rolling of leukocytes by reversible binding to carbohydrates (14). Thus, it is possible that Ptx decreases the leukocyte recruitment to the sites of inflamed tissue by competitively blocking common
Figure 5 - Pertussis toxin (Ptx) and dexamethasone (Dex) reduced the number of neutrophils in diapedesis in rat mesenteric tissue following exposure to LPS or fMLP. Ptx (1,200 ng/rat) and Dex $(0.2 \mathrm{mg} / \mathrm{rat})$ were injected iv $1 \mathrm{~h}$ before the ip administration of LPS (200 ng/cavity; A) or fMLP (44 ng/cavity; B). The rats were sacrificed $4 \mathrm{~h}$ after injection of the inflammatory stimuli. The neutrophils in diapedesis were counted in five consecutive microscopy fields along the venule. The results are reported as the mean \pm SEM of the number of neutrophils/field. Six rats were used per treatment. $* P<0.05$ compared to the group that received saline (S) iv (ANOVA and Tukey test).

Figure 6 - Pertussis toxin (Ptx) and dexamethasone (Dex) differentially affect the number of neutrophils inside the venule. Ptx (1,200 ng/rat) and Dex (0.2 $\mathrm{mg} / \mathrm{rat}$ ) were injected iv $1 \mathrm{~h}$ before the ip administration of LPS (200 ng/cavity; A) or fMLP (44 ng/cavity; B). The rats were sacrificed $4 \mathrm{~h}$ after injection of the inflammatory stimuli. The neutrophils were counted in five consecutive fields along the venule. The results are reported as the mean \pm SEM of the number of neutrophils in the lumen/ field. Six rats were used per treatment. $* \mathrm{P}<0.05$ compared to the group that received saline (S) iv (ANOVA and Tukey test). 
leukocyte and/or endothelial selectin carbohydrate binding sites (28). We have demonstrated that the in vivo anti-inflammatory effect of Ptx is dependent on its ability to inhibit the action of $\mathrm{G}_{\mathrm{i}}$ protein $(10,29)$. Furthermore, it has been shown that a Ptx-sensitive $\mathrm{G}$ protein is involved in cellular movement (2) as well as in other effects triggered by inflammatory mediators, including an oxidative burst, the release of arachidonatederived metabolites, and cell degranulation (30).

In conclusion, the histopathological procedure presented here proved to be an adequate means of quantifying the inflammatory and anti-inflammatory actions of several substances. The method showed a significant $(\mathrm{P}<0.05)$ correlation with the more conventional approach of leukocyte counts in peritoneal exudates. The two methods could not be strictly compared because of differences in the time course of neutrophil vascular migration to the mesentery (peak at the $3 \mathrm{rd} \mathrm{h}$ ) and the arrival of these cells into peritoneal cavities (peak at the 4th $h$ ). The principal advantage of this method compared to simple cell counting is the possibility of obtaining additional information about the steps involved in leukocyte migration. Such information may contribute to our understanding of the mechanisms underlying the action of different anti-inflammatory substances. For example, using this method it was possible to show that Ptx and Dex act via different mechanisms suggesting that Ptx prevents the locomotion of neutrophil from the vascular lumen into the interstitial space, whereas Dex acts at a step that precedes neutrophil arrival in the inflamed tissue, probably inhibiting production and/or activity of chemotactic agents. The methodology described here is not as practical as leukocyte counts in peritoneal exudates, but provides a good alternative to intravital microscopy $(31,32)$, because it is simpler and less expensive than the later technique, does not need prolonged training and can be carried out using the facilities of a conventional histology laboratory.

\section{Acknowledgments}

We thank Erik L. Hewlett for kindly providing the toxins and Stephen Hyslop for language review.

\section{References}

1. Springer TA (1994). Traffic signals for lymphocyte recirculation and leukocyte emigration: the multistep paradigm. Cell, 76: 301-304.

2. Ben-Baruch A, Michiel DF \& Oppenheim JJ (1995). Signals and receptors involved in recruitment of inflammatory cells. J ournal of Biological Chemistry, 270: 1170311706.

3. Nourshargh S, Larkin SW, Das A \& Williams TJ (1995). Interleukin-1-induced leukocyte extravasation across rat mesenteric microvessels is mediated by plateletactivating factor. Blood, 85: 2553-2558.

4. Frenette PS \& Wagner DD (1996). Adhesion molecules - Part II: Blood vessels and blood cells. New England J ournal of Medicine, 335: 43-45.

5. Gabe M (1968). Techniques Histologiques. Mason, Paris.
6. Souza GEP \& Ferreira SH (1985). Blockade by anti-macrophage serum of the migration of PMN neutrophils into the inflamed peritoneal cavity. Agents and Actions, 17: 97-103.

7. Arndt $H$, Palitzsch K-D, Anderson DC, Rusche J, Grisham MB \& Granger DN (1995). Leukocyte-endothelial cell adhesion in a model of intestinal inflammation. Gut, 37: 374-379.

8. Flower RJ (1989). Glucocorticoids and the inhibition of phospholipase $A_{2}$. In: Schleimer RP, Clamor HN \& Oronsky AL (Editors), Anti-Inflammatory Steroid Action. Basic and Clinical Aspects. Academic Press, New York, 46-66.

9. Imagawa $T$, Kanoh M, Sonoda S \& Utsumi S (1980). Polymorphonuclear leukocyteinhibitory factor of Bordetella pertussis. III. Inhibition of Arthus reaction and peri- toneal infiltration of PMN. Microbiology and Immunology, 24: 895-905.

10. Brito GCA, Souza MHLP, Melo-Filho AA, Hewlett EL, Lima AA, Flores CA \& Ribeiro RA (1997). Role of pertussis toxin A subunit in neutrophil migration and vascular permeability. Infection and Immunity, 65: 1114-1118.

11. Sindt KA, Hewlett EL, Redpath GT, Rappuoli R, Gray LS \& Vandenberg SR (1994). Pertussis toxin activates platelets through an interaction with platelet glycoprotein Ib. Infection and Immunity, 62: 3108-3114.

12. Katori M, Oda T \& Nagai K (1990). A site of action of dexamethasone on leukocyte extravasation in microcirculation. Agents and Actions, 29: 24-26.

13. Cybulsky MI, McComb DJ \& Movat HZ (1989). Protein synthesis dependent 
mechanisms of neutrophil emigration. Different mechanisms of inflammation in rabbits induced by interleukin-1, tumor necrosis factor alpha or endotoxins versus leukocyte chemoattractants. American J ournal of Pathology, 135: 227-237.

14. McEver RP (1992). Leukocyte-endothelial cell interactions. Current Biology, 4: 840849.

15. Flower RJ \& Blackwell GJ (1979). Antiinflammatory steroids induce synthesis of a phospholipase $A_{2}$ inhibitor which prevents prostaglandin generation. Nature, 278: 456-459.

16. Hirata $F$, Notsu $Y$, Iwata M, Parente L, DiRosa M \& Flower RJ (1982). Identification of several species of phospholipase inhibitory protein(s) by radioimmunoassay for lipomodulin. Biochemical and Biophysical Research Communications, 109: 223-230.

17. Staruch MJ \& Wood DD (1985). Reduction of serum interleukin-1 like activity after treatment with dexamethasone. J ournal of Leukocyte Biology, 37: 193-207.

18. Bochner BS, Rutledge BK \& Schleimer RP (1987). Interleukin 1 production by human lung tissue. II: inhibition by anti-inflammatory steroids. J ournal of Immunology, 139: 2303-2307.

19. Beutler B, Krochin N, Milsark IW, Luedke $C \&$ Cerami A (1986). Control of cachectin (tumor necrosis factor) synthesis: mechanisms of endotoxin resistance. Science, 232: 977-980.

20. Schwiebert LA, Beck LA, Stellato C, Bickel CA, Bochner BS \& Schleimer RP (1996).
Glucocorticoids inhibition of cytokine production: Relevance to antiallergic actions. J ournal of Allergy and Clinical Immunology, 97: 143-152.

21. Cronstein BN \& Weissmann G (1993). The adhesion molecules of inflammation. Arthritis and Rheumatism, 36: 147-157.

22. Tessier PA, Cattaruzzi $P \&$ \& McColl SR (1996). Inhibition of lymphocyte adhesion to cytokine-activated synovial fibroblasts by glucocorticoids involves the attenuation of vascular cell adhesion molecule 1 and intercellular adhesion molecule 1 gene expression. Arthritis and Rheumatism, 39: 226-234.

23. Dale DC, Fauci AS \& Wolff SM (1974). Alternate-day prednisone leukocyte kinetics and susceptibility to infections. New England J ournal of Medicine, 291: 11541158.

24. Ribeiro RA, Souza-Filho MVP, Souza MHLP, Oliveira SHP, Costa CHS, Cunha FQ \& Ferreira SH (1996). Role of resident mast cells and macrophages in the neutrophil migration induced by LTB4, fMLP and $\mathrm{C} 5 \mathrm{a}$ des Arg. International Archives of Allergy and Immunology, 112: 27-29.

25. Allison J r F, Smith MR \& Wood WB (1955). Studies on the pathogenesis of acute inflammation. II. The actions of cortisone on the inflammatory response to thermal injury. J ournal of Experimental Medicine, 102: 669-679.

26. Oda T \& Katori M (1992). Inhibition site of dexamethasone on extravasation of polymorphonuclear leukocytes in the hamster cheek pouch microcirculation. J ournal of
Leukocyte Biology, 52: 1337-1342.

27. Saukkonen K, Burnette WN, Mar V, Masur HR \& Tuomanen E (1992). Pertussis toxin has eukaryotic-like carbohydrate recognition domains. Proceedings of the National Academy of Sciences, USA, 89: 118-122.

28. Rodzinsky E, J ones $T$, Burnette WN, Burroughs M \& Tuomanen E (1993). Antiinflammatory effects in experimental meningitis of prokaryotic peptides that mimic selectin. J ournal of Infectious Diseases, 168: 1422-1428.

29. Thomazzi SM, Souza MHLP, Melo-Filho AA, Hewlett EL, Lima AAM \& Ribeiro RA (1995). Pertussis toxin from Bordetella pertussis blocks neutrophil migration and neutrophil-dependent edema in response to inflammation. Brazilian J ournal of Medical and Biological Research, 28: 120-124.

30. Nourshargh S \& Williams TJ (1990). Evidence that a receptor-operated event on the neutrophil mediates neutrophil accumulation in vivo. Pretreatment of $111 \mathrm{ln}$ neutrophils with pertussis toxin in vitro inhibits their accumulation in vivo. J ournal of Immunology, 145: 2633-2638.

31. Granger DN, Benoit J N, Suzuky M \& Grisham MB (1989). Leukocyte adherence to venular endothelium during ischemia/reperfusion. American J ournal of Physiology, 257: G683-G688.

32. House SD \& Lipowsky H (1987). Leukocyte-endothelium adhesion: microhemodynamics in mesentery of the cat. Microvascular Research, 34: 363-379. 